\title{
The Role of Neutral Hydrogen in the Evolution of Sprial and Irregular Galaxies
}

\author{
V.A. Ambartsumian ${ }^{1}$ and A.L. Gyulbudaghian \\ Byurakan Astrophysical Observatory, Armenia, e-mail \\ agyulb@bao.sci.am
}

The Hubble classification suggested for the spiral galaxies, $\mathrm{Sa}-\mathrm{Sb}-\mathrm{Sc}-\mathrm{Sm}-$ Irr, was considered by many astronomers as an evolution succession. The version of transformation from early spirals to the late spirals and vice versa was considered. The existence of several parameters, monotonically increasing or decreasing along this succession, together with the parameters which do not depend on the structural type of the galaxies of this succession, is a confirmation of this succession to be a succession of stages of evolution. Let us consider in detail both types of parameters. About the full masses and luminosities of galaxies we can state, that except Irr and maybe Sm-galaxies there is not a clear expressed difference of mean values of these parameters for galaxies of different structural types. Irr galaxies are the systems with low masses and luminosities, but on the (M-L) diagram a part of them is obviously mixed with the late spirals. There is a strong change of the $\mathrm{M} / \mathrm{L}$ ratio with changing of the structural type, though the early type galaxies on average apparently have the least values of that ratio. As monotonically changing parameters we can suggest the following three:

- Relative amount of $\mathrm{HI}(\mathrm{M}(\mathrm{HI}) / \mathrm{M}($ tot $))$.

- Relative amount of $\mathrm{HII}(\mathrm{M}(\mathrm{HII}) / \mathrm{M}$ (tot).

- The ratio of mass of $H_{2}$ to HI $\left(M\left(H_{2}\right) / M(H I)\right)$.

All these three parameters are changing monotonically along the succession $\mathrm{Sa}-$ Irr, moreover the third parameter is substantially decreasing (20 times), and the first and second are increasing correspondingly two times and several dozen times. These figures show, that along the succession Sa-Irr the quantity of $H_{2}$ is abruptly decreasing, and the quantity of $\mathrm{HI}$, on the contrary, is increasing. We think that as time elapses, the transformation of $H_{2}$ into HI takes place, that is why we must take Sa-Irr as a succession of evolution. Now the opposite point of view is widespread: the galaxies with high relative amount of $\mathrm{HI}$ are considered as an early phase of evolution of spiral galaxies. It is also assumed that the clouds of two other types $\left(\mathrm{H}_{2}\right.$ and HII) must be formed from the HI clouds. We have here an analog of a long existing dilemma: the transformation of diffuse matter into the more dense bodies (nobody observed such a process) or the transformation of more dense objects into dilute matter, and the first concept is more widespread than the second, which has direct observational confirmations. The question of direction of evolution from the molecular clouds

${ }^{1}$ Deceased 
to the clouds of HI is one of the components of a larger question about the direction of evolution of galaxies. Let us summarise the results of observations, presented in the literature.

- There is a clear increase of relative amount of HI along the succession $\mathrm{Sa}-\mathrm{Sb}-\mathrm{Sc}-\mathrm{Sm}-\mathrm{Irr}$.

- Many irregular and some Sc galaxies have large HI envelopes.

- There are dense HI intergalactic clouds, some of them are $4 \times 10^{9}$ years old (in Leo) and have the masses of dwarf galaxies.

- The $\mathrm{H}_{2}$ clouds, as well as HI clouds are concentrated towards the spiral arms (in our Galaxy, as well as in other galaxies), but the arrangement along the spiral arms in $\mathrm{CO}$ is more clear, than in $\mathrm{HI}$.

- We have a case of transformation of molecular clouds into HI clouds within the galaxy M83 (under the influence of bright stars radiation).

- The investigation of radial systems of dark globules in our Galaxy shows, that a transformation of $\mathrm{H}_{2}$ clouds into $\mathrm{HI}$ clouds takes place (with an intermediate stage of $\mathrm{HII})$.

The first two points are possible to interprete from the point of view of transformation of $\mathrm{H}_{2}$ into $\mathrm{HI}$ and vice versa (we have then for the evolution of galaxies correspondingly $\mathrm{Sa}-\mathrm{Sb}-\mathrm{Sc}-\mathrm{Sm}$-Irr or vice versa), but the other points are clearly in favour of the transformation of $\mathrm{H}_{2}$ clouds into $\mathrm{HI}$ clouds. It means that the older galaxies have higher values of the ratio $\mathrm{M}(\mathrm{HI}) / \mathrm{M}$ (tot). We have the following observational data (from the literature), about clusters of galaxies.

- When we are approaching the center of a cluster, the deficit of HI in the spiral galaxies is increasing.

- The deficit of HI is higher in the cluster galaxies than at the field galaxies.

- The clusters having high $\mathrm{X}$-ray radiation have also more galaxies with $\mathrm{HI}$ deficit.

Taking into account the preceding three points, we can make the following conclusion: the galaxies situated in the younger systems have also higher HI deficit. We can make three main conclusions from the whole paper:

- In our Galaxy, as well as in other spiral galaxies, a transformation of $\mathrm{H}_{2}$ clouds into HI clouds takes place.

- The older the galaxy, the higher the percentage of HI quantity (in some cases almost the whole galaxy can consist of $\mathrm{HI}$ ).

- The evolution path for the spiral galaxies passes from the early spirals to the irregular galaxies. 